\title{
NEOPLASIA TROFOBLÁSTICA GESTACIONAL: HOSPITAL FÉLIX BULNES CERDA, 1992 - 2002.
}

\author{
Sergio Aitken S. ${ }^{1}$, Alicia Benavides M. ${ }^{2}$, Marcia Smirnow S. ${ }^{1}$ \\ ${ }^{1}$ Servicio de Obstetricia y Ginecología, Hospital Félix Bulnes Cerda. ${ }^{2}$ Servicio de Anatomía Patológica, Hospital Félix \\ Bulnes Cerda.
}

\section{RESUMEN}

Objetivo: Analizar los hallazgos clínicos y el seguimiento de los casos de neoplasia trofoblástica gestacional. Material y Métodos: Se revisaron todos los casos de mola hidatidiforme en el Hospital Félix Bulnes Cerda, entre los años 1992 y 2002. Resultados: Se diagnosticaron 79 casos. La edad promedio de las pacientes fue 26,4 años. El diagnóstico de mola hidatidiforme fue sospechado clínicamente en $75,6 \%$ y en $24,4 \%$ fue hallazgo anatomopatológico. Los principales síntomas al ingreso fueron: dolor hipogástrico $(75,6 \%)$, náuseas y/o vómitos $(32,1 \%)$ y disuria $(19,2 \%)$. Entre los signos clínicos destacan sangrado vaginal $(83,3 \%)$, altura uterina discordante $(25,3 \%)$ y expulsión de vesículas $(12,8 \%)$. La ecografía concluyó mola hidatidiforme en $74,4 \%$ y quistes tecaluteínicos en $23,1 \%$. Los hallazgos de anatomía patológica fueron mola completa $(61,5 \%)$, mola parcial $(31,2 \%)$ y coriocarcinoma $(1,3 \%)$. En el seguimiento, $30,8 \%$ no volvieron a control y $23,1 \%$ tuvieron entre 7 y 12 controles. Conclusión: Los hallazgos de este estudio son similares a los descritos en la literatura.

\section{PALABRAS CLAVES: Mola hidatidiforme, enfermedad trofoblastica gestional}

\section{SUMMARY}

Objective: To analyze the clinical findings and the follow-up of gestational trophoblastic neoplasia. Material and Methods: All cases of molar pregnancy at Felix Bulnes Hospital, Santiago de Chile, between 1992 and 2002 were reviewed. Results: 79 cases were found. The average age of patients was 26.4 years. The diagnosis of hydatidiform mole was clinically suspected in $75.6 \%$, in the rest it was an anatomopathological finding. The main symptoms at admission were: hypogastric pain $(75.6 \%)$, nausea and / or vomiting (32.1\%) and dysuria (19.2\%). The clinical signs were: vaginal bleeding (83.3\%), discordant uterine height $(25.6 \%)$ expulsion of vesicles (12.8\%). Ultrasound concluded hydatidiform mole in $75.4 \%$ and tecaluteinic cysts in $23.1 \%$. Anatomopathological diagnose were: complete mole $(61.5 \%)$, partial mole (37.1\%) and choriocarcinoma (1.3\%). Follow-up: $30.8 \%$ didn't come to control, and $23.1 \%$ has between 7 and 12 controls. Conclusion: Our finding denote frequent characteristics observed in the literature description.

\section{KEY WORDS: Hydatidiform mole, gestational trophoblastic disease}

\section{INTRODUCCIÓN}

La enfermedad trofoblástica gestacional comprende una amplia gama de lesiones que van desde alteraciones benignas como la mola hidatidiforme hasta malignas como el coriocarcinoma.

Existe una importante variación con respecto 
a la incidencia en distintos lugares del mundo, desde 1 en 1000 a 1500 partos en Estados Unidos hasta 10 en 1000 partos en Indonesia (1). En Chile la incidencia es alrededor de 1 por 1000 partos $(2,3)$.

El objetivo de este estudio es evaluar el diagnóstico, tratamiento y seguimiento de la neoplasia trofoblástica gestacional, manejadas en el Hospital Félix Bulnes Cerda en el período $1992-2002$.

\section{MATERIAL Y MÉTODO}

Se realizo un estudio descriptivo retrospectivo a partir de la revisión de los informes de anatomía patológica de pacientes con diagnóstico de embarazo molar en el Hospital Felíx Bulnes Cerda, en el período comprendido entre los años 1992-2002. A partir de esos informes se ubicaron las fichas clínicas para su revisión. Las variables consideradas fueron: edad de la paciente, tiempo de amenorrea, paridad, hallazgos ultrasonográficos, hallazgos clínicos, diagnóstico anatomopatológico, número de controles post evacuación.

\section{RESULTADOS}

De los 79 casos diagnosticados, en 60 el diagnóstico de embarazo molar fue al menos sospechado por algún hallazgo, ya sea clínico o de laboratorio (75,6\%). En los casos restantes, 19 $(24,4 \%)$ el diagnóstico inicial fue restos de aborto o aborto incompleto, y el diagnóstico de enfermedad del trofoblasto fue anatomopatológico.

La edad promedio fue de 26,1 años (rango: 14 y 50 años), con mayor frecuencia en los extremos de la vida reproductiva (25 de los 79 casos ocurrieron antes de los 20 y después de los 40 años).

La mayor cantidad de casos fueron encontrados en multíparas $(69,2 \%)$ que en primíparas $(30,8 \%)$. Los síntomas y signos más frecuentes hallados en la historia clínica se presentan en la Tabla I.

La ecografía concluyó mola hidatidiforme en $75,4 \%$ y quistes tecaluteínicos en $23,1 \%$. El diagnóstico anatomopatológico se presenta en la Tabla II.

Después del vaciamiento, las pacientes en su mayoría siguieron en control; un grupo importante de pacientes no volvió a seguimiento (Tabla III).

El descenso de la curva de gonadotropina coriónica humana fue normal en todos los casos
Tabla I

NEOPLASIA TROFOBLÁSTICA GESTACIONAL. SÍNTOMAS Y SIGNOS.

\begin{tabular}{lrr}
\hline Síntomas y signos & $n$ & $\%$ \\
\hline Dolor hipogástrico & 59 & 75,6 \\
Náuseas y / o vómitos & 55 & 32,1 \\
Disuria & 15 & 19,2 \\
Sangrado vaginal & 65 & 83,3 \\
Altura uterina discordante & 20 & 25,6 \\
Expulsión de vesículas & 10 & 12,8 \\
\hline
\end{tabular}

Tabla II

NEOPLASIA TROFOBLÁSTICA GESTACIONAL. ANATOMÍA PATOLÓGICA.

\begin{tabular}{lrr}
\hline Diagnóstico & $n$ & $\%$ \\
\hline Mola completa & 48 & 61,5 \\
Mola parcial & 29 & 37,2 \\
Coriocarcinoma & 1 & 1,3 \\
\hline
\end{tabular}

Tabla III

NEOPLASIA TROFOBLÁSTICA GESTACIONAL. FRECUENCIA DE CONTROLES POST EVACUACIÓN.

\begin{tabular}{lrr}
\hline$N^{\circ}$ controles & $n$ & $\%$ \\
\hline 0 & 24 & 30,8 \\
1 & 12 & 15,4 \\
2 & 10 & 12,3 \\
3 & 4 & 5,1 \\
5 & 2 & 2,6 \\
6 & 8 & 10,3 \\
7 & 2 & 2,6 \\
8 & 10 & 12,8 \\
9 & 1 & 1,3 \\
10 & 3 & 3,8 \\
12 & 2 & 2,6 \\
\hline
\end{tabular}

excepto en 2, que fueron derivados a quimioterapia. El único caso de coriocarcinoma correspondió a una hemorragia persistente del postparto, en el que se encontró además una anemia severa y un útero aumentado de tamaño y fue derivada a quimioterapia.

\section{DISCUSIÓN}

La mola hidatidiforme es una patología que debe estar en la mente del clínico a pesar de no ser tan frecuente, principalmente por el potencial 
maligno del coriocarcinoma y el hecho de que $24,4 \%$ de los casos de nuestro estudio no fueron sospechados por el médico tratante y solo fue un hallazgo anatomopatológico. En esta revisión, las características clínicas coinciden con las señaladas por la literatura, así como la mayor frecuencia en las edades extremas de la vida. En cuanto al seguimiento, llama la atención que $30,8 \%$ de los casos no tuvieron seguimiento posterior; esto puede deberse a que en $24,4 \%$ de los casos el diagnóstico de mola fue un hallazgo anatomopatológico, por lo que el contacto con la paciente fue hecho bastante tiempo después.

En cuanto a la edad, esta patología es más común en los extremos de la edad reproductiva, siendo más frecuente en mujeres mayores de 40 años $(4,5)$ y en menores de $20(6)$, esto también fue observado en nuestra experiencia ya que 25 de los 79 casos ocurrieron antes de los 20 y después de los 40 años.

Las características clínicas son genitorragia la que casi siempre está presente; en nuestra casuística $83,3 \%$ de las pacientes presentaron este síntoma. La hiperemesis gravídica es un síntoma muy frecuente comprometiendo casi a un tercio de las pacientes $(32,1 \%)$. La hipertensión arterial no fue un hecho común en nuestra experiencia y probablemente se deba al diagnóstico ultrasonográfico precoz que permitió la interrupción oportuna del embarazo. El hipertiroidismo descrito en $7 \%$ de los casos (7) no fue observado en nuestra experiencia. También se describen la expulsión de vesículas por vía vaginal, hemorragias persistentes del postparto o derivado de metástasis en distintos parénquimas (8).

En el examen físico destaca una paciente más o menos comprometida (según el grado de anemia), útero de tamaño mayor a la amenorrea, tumores ováricos bilaterales; estos últimos corresponden a quistes tecaluteínicos encontrados en $23,1 \%$ de las pacientes. Para el diagnóstico es necesario el estudio ultrasonográfico, donde se describe la imagen clásica de "panal de abeja", en la mayoría de los casos la ecografía facilita el diagnóstico precoz antes del inicio de los síntomas y permite reducir la morbimortalidad materna.

Al confirmar el diagnóstico, debe realizarse el vaciamiento uterino completo mediante aspiración y/o legrado uterino instrumental. Debe considerarse también la posibilidad de histerectomía en mujeres que no desean más hijos.
Sin embargo, ni la evacuación ni la histerectomía significa el término de la enfermedad, por el riesgo de persistencia y enfermedad metastásica. El $80-90 \%$ de las molas son benignas y no dan más problemas, el $10 \%$ progresa a mola invasora y $2,5 \%$ a coriocarcinoma $(8,9)$.

El control y seguimiento debe hacerse con determinaciones seriadas de subunidad beta de $\mathrm{GCH}$, cada dos semanas hasta la negativización de dos valores consecutivos y luego mensualmente hasta completar 6 meses; la mayoría de las pacientes negativizan los niveles antes de las 12 semanas post vaciamiento (10). El seguimiento debe ser idealmente hasta el año, y es importante evitar una gestación por que elevaría los niveles de $\mathrm{GCH}$, marcador tumoral fundamental para el seguimiento (7).

En nuestra experiencia 2 de 79 casos requirieron quimioterapia $(2,5 \%)$ por enfermedad del trofoblasto persistente, menor al $13,4 \%$ y similar en frecuencia de coriocarcinoma $(1,3 \%)$ con otra experiencia nacional (11).

El tratamiento es motivo de controversia dado los múltiples esquemas terapéuticos debido a la falta de estandarización para la clasificación y de criterios que dificulta la comparación entre las series $(12,13,14)$. En 2000 se propuso la nueva clasificación de riesgo que permitirá estandarizar los conceptos para el tratamiento de la enfermedad $(16,17)$.

\section{BIBLIOGRAFÍA}

1. Bracken MB, Brinton LA, Hayashi K. Epidemiology of hydatidiform mole and choriocarcinoma. Epidemiol Rev 1984; 6:52-60.

2. Barcellos JM, Belfort P, De Rezende J. Enfermedad trofoblástica (neoplasias trofoblásticas gestacionales) En: Pérez Sánchez A (ed): Obstetricia. Cap.26 Santiago, Chile: Publicaciones Técnicas Mediterráneo, 1985; 326-330.

3. Cruzat L, Mayerson D. Wild R, Fernández M. Neoplasia trofoblástica gestacional. Rev Chil Obstet Ginecol 1985; 2:95-106.

4. Copeland L, Jarrel J, Mc Gregor J. Textbook of Gynecology. 1993.

5. Buckley JD. The epidemiology of molar pregnancy and choriocarcinoma. Clin Obstet Gynecol 1984; 27: 193.

6. Slocumb JC, Lund CJ. Incidence of trophoblastic disease: increased rate in youngest age group. Am J Obstet Gynecol 1969; 104: 421.

7. Wild R, Mayerson D, Cuello M. Neoplasia trofoblástica gestacional. Enfermedad del trofoblasto. 
En: Obstetricia. Pérez A, Donoso E (ed). Editorial Mediterráneo. Tercera Edición. 1999, 462-471.

8. Lurain JR, Brewer JI, Torok EE, Halpern B. Natural history of hydatidiform mole after primary evacuation. Am J Obstet Gynecol 1983; 145: 591.

9. Pacheco J, Alegre J, Paniagua G. Características clínicas y anatomopatológica en mola hidotidiforme: Anales de la Facultad de Medicina, Universidad de San Marcos 2002; 63(4): 275 - 280.

10. Goycolea JP, Cuello M, Mayerson D, Brañes J. Neoplasia trofoblástica gestacional (parte I): criterios de diagnóstico. Rev Chil Obstet Ginecol 2003; 68(3): $248-255$.

11. Mayerson D, Cuello M, Cartens M, Chuaqui R, Badía J, Wild R. Manejo de la enfermedad del trofoblasto: mola parcial y completa. Rev Chil Obstet Ginecol 1999; 64(6): 477-485.

12. Bagshawe KD, Dent J, Newlands ES. The role of low-dose methotrexate and folinic acid in gestacional trophoblastic tumour (GTT). Br J Obstet Gynaecol 1989; 96(7): 795-802.
13. Garrett AP, Garner EO, Goldstein DP. Methotrexate infusion and folinic acid as primary therapy for nonmetastatics and low-risk metastatics gestational trophoblastic tumor. 15 years of experience. J Reprod Med 2002; 47(5): 355-362.

14. Homesley HD, Blessing JA, Rettenmaier M. Weekly intramuscular methotrexate for nonmetastatic gestational trophoblastic disease. Obstet Gynecol 1988; 72(3 Pt 1): 413-418.

15. Wong LC, Choo YC, Ma HK. Methotrexate with citrovorum factor rescue in gestational trophoblasticdisease. Am J Obstet Gynecol 1985; 152(1): 59-62.

16. Kohorn El. The new FIGO 2000 staging and risk factor scoring system for gestational trophoblastic disease: description and critical assessment. Int $\mathrm{J}$ Gynecol Cancer2001; 11(1): 73-77.

17. Kohorn El. Negotiating a staging and risk factor scoring system for gestational trophoblastic neoplasia. A progress report. J Reprod Med 2002; 47(6): 445-450. 